\title{
834 A ROBUST DEEP LEARNING APPROACH FOR PRECISELY SEGMENTING CELLS IN MULTIPLEX TISSUE IMAGES
}

${ }^{1}$ Daniel Winkowski, ${ }^{1}$ Jeni Caldara, ${ }^{1}$ Brit Boehmer, ${ }^{2}$ Regan Baird*. 'Visiopharm, Westminster, CO, USA; ${ }^{2}$ Visiopharm Corp, Roanoke, VA, USA

Background Multiplex images are becoming pivotal in tissue pathology because they provide positional location and multidimensional phenotype of every cell. The heterogeneity of cells, morphologies, and densities makes the identification of the millions of cells in a tissue slice challenging. There is an urgent need for a robust, yet flexible, algorithm to automatically demarcate each cell that accurately defines cellular boundaries. We have developed a method to extend a DL nuclear identification algorithm beyond the nucleus and to the outer boundary of the cell using biological signals from multiplex panels.

Methods All image analysis was performed in the Visiopharm image analysis platform. Three human observers provided ground truth (GT) annotations by outlining cells in predefined areas each containing $\sim 30$ cells in six different images from two different multiplex instruments: $\mathrm{mIF}=8$-plex via Vectra Polaris from Akoya and IMC $=13$-plex via Hyperion from Fluidigm. Images were subsequently segmented by different AI methods: Machine Learning Nuclear Detection (ML), Deep Learning Nuclear Detection (DL), and DL that incorporates biological signals $(\mathrm{DL}+)$. Each set of computer-generated annotations was compared to GT using common evaluation metrics DICE, Precision and Sensitivity.

Results Overall, we found a high degree of concordance between the computer-generated and human annotations (DICE $=0.73 \pm 0.08, \mathrm{n}=12)$ and between imaging modalities (mIF: $0.76 \pm 0.07$; IMC: $0.71 \pm 0.08 ; \mathrm{n}=6$ ). Comparison of DICE scores for the AI methods indicated a superior delineation of cell boundaries using the DL+ method (DL+: 0.79 \pm 0.07 ; ML: $0.74 \pm 0.08$; DL: $0.74 \pm 0.03$;). Precision, which compares true vs false positive annotated regions to GT, was also high for all images $(0.77 \pm 0.11)$ (mIF: $0.76 \pm 0.10$; IMC: $0.78 \pm 0.11$ ). Sensitivity, which compares true positives vs false negative annotated regions GT, was also high for all images

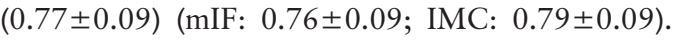

Conclusions We developed a flexible DL based strategy that enables the most comprehensive segmentation of cells in multiplex tissue images. Each AI approach shows a high concordance with segmentation annotations from human observers as measured by the industry standards DICE, Precision and Sensitivity. The DL + method did achieve the highest DICE score indicating a more accurate delineation of cell boundaries. Expectedly, precision and sensitivity metrics are similar between all methods while DICE Coefficient better accounts for the annotations at the cell edge. The DL+ cell segmentation algorithm will yield an improved accuracy when phenotyping cells in downstream analysis as the precise biomarker composition is more accurately contained within each cell.

http://dx.doi.org/10.1136/jitc-2021-SITC2021.834 